\title{
Editorial
}

\section{Sleep In Pregnancy and the Postpartum Period}

\author{
Louise M. O’Brien*
}

Sleep Disorders Center, University of Michigan, Med Inn Building Rm C736, 1500 E. Medical Center Drive, Ann Arbor, MI 48109-5845, USA

Sleep disturbance is common in pregnant women and an increasing number of studies supports a relationship between sleep disturbance and poor pregnancy outcomes. Such outcomes may not only be limited to the health of the mother, such as gestational hypertension and pre-eclampsia, but also to the health of the fetus, such as growth restriction. Despite these findings few obstetric healthcare providers screen their patients for sleep problems. This may be due to the belief that poor sleep in pregnancy is a natural part of the transition to parenthood and is in preparation for the loss of parental sleep that accompanies a newborn, or that the sleep difficulties are mostly transient and get resolved following delivery. Parental sleep difficulties may persist or emerge following delivery and may be associated with mood disorders and family dysfunction. Clearly this has an impact on the wellbeing of the offspring.

In this Special Issue on Sleep in Pregnancy and the Postpartum Period, a range of maternal sleep problems during pregnancy is highlighted. Although short sleep duration is recognized as a risk factor for adverse health conditions, Okun and colleagues demonstrate how long sleep duration in early pregnancy contributes to low-grade inflammation. They discuss how this may initiate or augment pathophysiological changes associated with morbidity. Facco discusses the evidence regarding a role for maternal sleep disruption, including sleep duration as well as habitual snoring, in glucose control and gestational diabetes. Furthermore, sleep disruption in the non-pregnant population is a known independent risk factor for cardiovascular disease. One of the most robust findings in the literature is that of the strong association between maternal sleep disordered breathing and gestational hypertension/pre-eclampsia. In this issue, Bourjeily and colleagues provide an excellent overview of how maternal sleep-disordered breathing could be linked to hypertensive disorders of pregnancy and poor pregnancy outcomes, including a detailed discussion of potential mechanistic pathways. Moreover, Tantrakul and colleagues suggest that positive airway pressure may be potential treatment for preeclampsia.

\footnotetext{
*Address correspondence to this author at the Sleep Disorders Center, University of Michigan, Med Inn Building Rm C736, 1500 E. Medical Center Drive, Ann Arbor, MI 48109-5845, USA;

Tel: 734-763-9684; E-mail: louiseo@med.umich.edu
}

In addition to gestational hypertension/pre-eclampsia and gestational diabetes, the presence of sleep-disordered breathing during pregnancy raises other potential concerns including maintenance of an adequate airway in women undergoing anesthesia, particularly in those women general anesthesia is required for operative or urgent deliveries. The perspective of an anesthesiologist is provided by Bullough, who discusses clinical anesthetic management of the high risk parturient with sleep-disordered breathing. Although breathing disorders comprise much of the literature, other sleep difficulties are also prevalent during pregnancy. One such condition is restless legs syndrome, which is particularly common in pregnancy but receives little attention. Hensley and Shamim-Uzzaman provide an overview of restless legs syndrome and discuss the prevalence, etiologies, diagnosis, and treatment of this condition and also provide helpful education for the clinician. Along with maternal outcomes, poor sleep during pregnancy may also impact fetal outcomes. Tauman provides an overview of the limited literature to date that has investigated fetal wellbeing in women with sleep disruption during the prenatal period, particularly sleep-disordered breathing.

The birth of a baby also brings about additional challenges to maternal sleep. While several sleep problems may be reduced following delivery, frequent sleep disturbances in order to feed or attend to a newborn affect almost all parents. Russell and colleagues provide a critical, contextual and evolutionary perspective on recent evidence surrounding the development of normal infant sleep in the postnatal period. They discuss sleep in relation to feeding method, sleep location, and the associations with Sudden Infant Death Syndrome. Infant sleep location is a controversial issue and many parents are advised to avoid bed sharing with their young infant. Stremler and colleagues discuss recommendations for infant sleep location and the effects of this choice on both maternal sleep and other health outcomes. They provide data from a randomized controlled trial of a sleep intervention for mothers and their newborns. Following Stremler, Montgomery-Downs and colleagues show that postpartum sleep disturbance and fatigue are major contributing factors to the onset of mood disturbance which in turn is strongly associated with an increase in negative infant-parent interactions and adverse infant emotional and cognitive outcomes. Of particular interest, and in addition to maternal sleep disruption, these authors discuss paternal 
sleep. Little research has involved sleep disruption in new fathers yet fathers play an equal role in the health and wellbeing of a family unit, and consequently optimal infant development. Finally, data are presented by Swanson and colleagues who used focus groups to explore sleep and mood during the postpartum period in women at risk for postpartum depression. The themes raised by these women suggest areas of potential focus for future studies with larger perinatal samples.

(C) Louise M. O'Brien; Licensee Bentham Open.

This is an open access article licensed under the terms of the Creative Commons Attribution Non-Commercial License (http://creativecommons.org/licenses/by$\mathrm{nc} / 3.0 /$ ), which permits unrestricted, non-commercial use, distribution and reproduction in any medium, provided the work is properly cited. 